\title{
Impairment of intestinal glutathione synthesis in patients with inflammatory bowel disease
}

\author{
B Sido, V Hack, A Hochlehnert, H Lipps, C Herfarth, W Dröge
}

\begin{abstract}
Background-Reactive oxygen species contribute to tissue injury in inflammatory bowel disease (IBD). The tripeptide glutathione (GSH) is the most important intracellular antioxidant.

Aims-To investigate constituent amino acid plasma levels and the GSH redox status in different compartments in IBD with emphasis on intestinal GSH synthesis in Crohn's disease.

Methods-Precursor amino acid levels were analysed in plasma and intestinal mucosa. Reduced (rGSH) and oxidised glutathione (GSSG) were determined enzymatically in peripheral blood mononuclear cells (PBMC), red blood cells (RBC), muscle, and in non-inflamed and inflamed ileum mucosa. Mucosal enzyme activity of $\gamma$-glutamylcysteine synthetase $(\gamma \mathrm{GCS})$ and $\gamma$-glutamyl transferase $(\gamma \mathbf{G T})$ was analysed. Blood of healthy subjects and normal mucosa from a bowel segment resected for tumour growth were used as controls.
\end{abstract}

Results-Abnormally low plasma cysteine and cystine levels were associated with inflammation in IBD $\left(p<10^{-4}\right)$. Decreased rGSH levels were demonstrated in noninflamed mucosa $(p<0.01)$ and inflamed mucosa $\left(p=10^{-6}\right)$ in patients with IBD, while GSSG increased with inflammation $(p=0.007)$ compared with controls. Enzyme activity of $\gamma$ GCS was reduced in non-inflamed mucosa $(p<0.01)$ and, along with $\gamma$ GT, in inflamed mucosa $\left(p<10^{-4}\right)$. The GSH content was unchanged in PBMC, RBC, and muscle.

Department of

Surgery, University of Heidelberg,

Heidelberg, Germany B Sido

A Hochlehnert

C Herfarth

Department of Immunochemistry, German Cancer Research Center, Heidelberg, Germany

V Hack

H Lipps

W Dröge

Correspondence to: Dr B Sido, Department of Surgery, University of

Heidelberg, Im Neuenheimer Feld 110, 69120 Heidelberg, Germany.

Accepted for publication 31 October 1997 enzymes involved in GSH synthesis accompanied by a decreased availability of cyst(e)ine for GSH synthesis contribute to mucosal GSH deficiency in IBD. As the impaired mucosal antioxidative capacity may further promote oxidative damage, GSH deficiency might be a target for therapeutic intervention in IBD.

(Gut 1998;42:485-492)

Keywords: Crohn's disease; ulcerative colitis; glutathione; amino acids; $\gamma$-glutamylcysteine synthetase; mucosa

Infiltrating macrophages and neutrophils are abundantly present in inflamed gut in patients with inflammatory bowel disease (IBD). There is a growing body of evidence that these cells expose the inflamed intestine to substantial oxidative stress by production of reactive oxygen species (ROS). ${ }^{1-4}$ Excessive production of ROS could be also demonstrated for circulating phagocytic cells in patients with $\mathrm{IBD}^{5}$ and was shown to be involved in several models of experimental colitis. ${ }^{6-8}$ The mucosa is endowed with various endogenous antioxidant defence systems to remove ROS resulting from normal metabolism. However, enzyme activities of catalase, superoxide dismutase, and glutathione peroxidase were reported to be low at only $4 \%, 8 \%$, and $45 \%$, respectively, in healthy colonic mucosa compared with the liver. ${ }^{9}$ ROS are highly toxic to cells and oxygen radical formation in excess of physiological amounts may overtax the limited intestinal antioxidant defence system initiating oxidative injury to the gut. ${ }^{10}{ }^{11}$ Deterioration of antioxidative glutathione metabolism ${ }^{12}$ and increased colonic oxidative damage to proteins and DNA in association with impaired enzyme activity of CuZn superoxide dismutase has been reported previously in patients with Crohn's disease (CD) ${ }^{13}$ Efficacy of current standard therapy in IBD was suggested to be related to antioxidant actions: 5 -aminosalicylic acid is a highly potent scavenger of $\operatorname{ROS}^{14}$ and was shown to reduce lipid peroxidation in $\mathrm{CD}$ and ulcerative colitis (UC) ${ }^{15}$ Preliminary uncontrolled clinical trials showed that $\mathrm{CuZn}$ superoxide dismutase may be of benefit in $\mathrm{CD}^{16}$ and UC. ${ }^{17}$ Several classical antioxidants were reportedly effective in reducing ROS production in $\mathrm{UC}^{18}$ and experimental colitis. ${ }^{78}$ Intestinal inflammation induced by administration of an ROS-generating agent in rats could be reversed by supplementation of glutathione..$^{19}$ It has therefore been proposed that IBD results from an imbalance between pro-oxidant and antioxidant mechanisms. ${ }^{20} 21$

The tripeptide glutathione (L- $\gamma$-glutamyl-L- cysteinylglycine, GSH) is the most important intracellular antioxidant. In the presence of ROS reduced GSH (rGSH) is oxidised to glutathione disulphide (GSSG). This reaction is catalysed by GSH peroxidase and reversed by GSH reductase (fig 1). ${ }^{22}$ The intestinal GSH content very much depends on GSH synthesis of $\gamma$-glutamylcysteine synthetase $(\gamma$ GCS) results in a rapid decrease of GSH within 30 minutes, ultimately leading to mucosal damage after several days. ${ }^{23} \gamma \mathrm{GCS}$, the rate limiting enzyme in GSH synthesis (labelled 2 in fig1), is controlled via a feedback mechanism by the end product GSH. ${ }^{24}$ High intracellular glutamate levels support GSH synthesis as glutamate competes with GSH at the regulatory site of the enzyme $\gamma$ GCS.$^{24}$ However, high extracellular glutamate concentrations impair GSH as in vivo administration of a specific inhibitor 


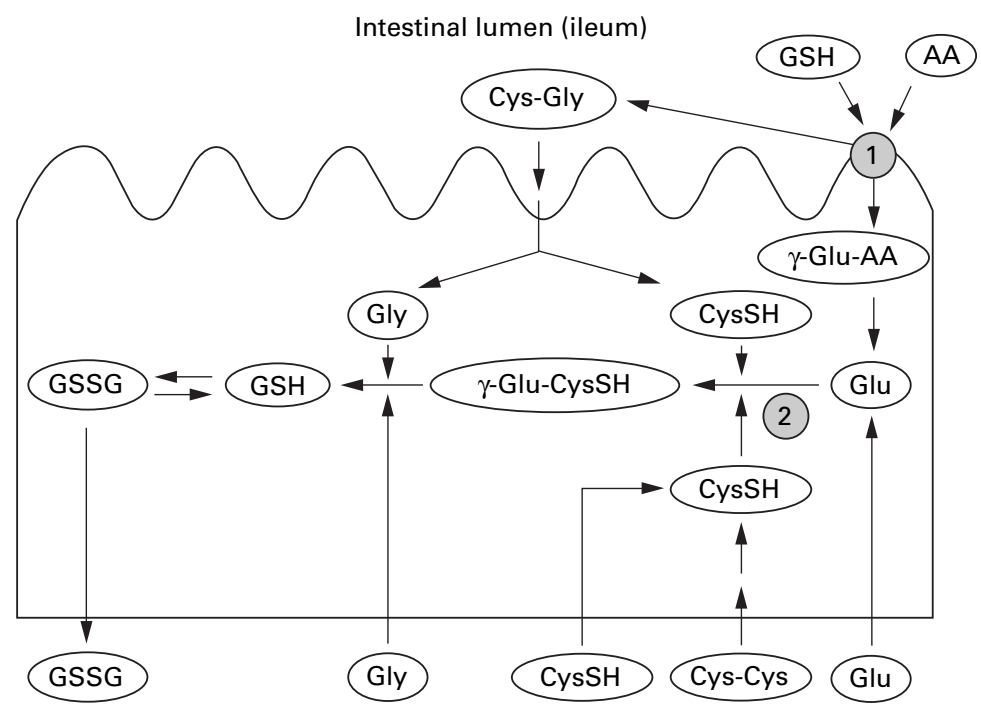

Plasma

Figure 1 Simplified diagram of mucosal glutathione synthesis in the ileum. GSH, glutathione; GSSG, glutathione disulphide; Gly, glycine; CysSH, cysteine; Cys-Cys, cystine; Glu, glutamate; $A A$, amino acid; $\gamma$-Glu-AA, $\gamma$-glutamyl-amino acid; $\gamma$-Glu-Cys $S H$, $\gamma$-glutamylcysteine; Cys-Gly, cysteinylglycine. (1) Site of action of $\gamma$-glutamyl transferase; (2) site of action of $\gamma$-glutamylcysteine synthetase. For further information see introduction.

synthesis by competitively inhibiting intracellular uptake of cystine ${ }^{25}$ through the commonly used membrane transport system. ${ }^{26}$ The amino acids cysteine and its disulphide cystine are limiting precursors of GSH synthesis. ${ }^{22} 27$ Alternatively, luminal GSH is degraded via the $\gamma$-glutamyl transferase $(\gamma \mathrm{GT})$ reaction to $\gamma$-glutamyl amino acids that are transported to the cell to be used for resynthesis of GSH (labelled 1 in fig 1). ${ }^{28}$ Under physiological conditions uptake of intact GSH across the brush border membrane seems to be relevant for the jejunum only. ${ }^{29}{ }^{30}$ In the present study we evaluated the GSH level and redox status in different compartments in patients with IBD and focused on intestinal GSH synthesis by determining the concentration of the precursor amino acids in the plasma and ileum mucosa, as well as the mucosal enzyme activity of $\gamma \mathrm{GCS}$ and $\gamma \mathrm{GT}$.

\section{Methods}

PATIENTS

Thirty three patients with CD (16 men and 17 women) and 33 patients with UC (19 men and 14 women) with a mean age of 37 (range 19-65) years undergoing resection of inflamed bowel in CD or restorative proctocolectomy with ileal pouch-anal anastomosis in UC were included in the study. None of the patients received parenteral nutrition during a period of three months before the operation. All patients were free of intestinal inflammation postoperatively. IBD was histologically verified by a qualified pathologist. Clinical disease activity was evaluated according to Best for CD patients (CDAI) ${ }^{31}$ and according to Rachmilewitz for UC patients. ${ }^{32}$ In five patients with CD and four with UC the clinical activity index could not be calculated because of pre-existing oostomy. No N-acetyl-L-cysteine was administered throughout the study period. Steroid treatment did not have a significant influence on any of the parameters tested either in blood or tissue, so the data of patients with and without steroids were pooled. Permission to perform the study was obtained from the local ethics committee.

PROCESSING OF SAMPLES

Heparinised venous blood was taken from fasting patients preoperatively, and 10 days and three months after the operation and was kept on ice until centrifugation (within 20 minutes). Plasma was deproteinised with $2.5 \%$ sulphosalicylic acid (SSA), incubated for 10 minutes at $4^{\circ} \mathrm{C}$, and subjected to centrifugation (12 $000 \mathrm{~g}, 10$ minutes). The supernatant was stored at $-80^{\circ} \mathrm{C}$ until use. Peripheral blood mononuclear cells (PBMC) were isolated on a Ficoll gradient (Lymphoprep, Nycomed Pharma, Oslo, Norway), lysed in $0.4 \mathrm{ml} 2.5 \%$ SSA $\left(10\right.$ minutes, $\left.4^{\circ} \mathrm{C}\right)$, and centrifuged. The supernatant was stored at $-80^{\circ} \mathrm{C}$ until assay of GSH. Red blood cells (RBC) from $0.1 \mathrm{ml}$ blood were processed as described for PBMC. For biochemical analysis of mucosa only patients with CD were considered because of the long ischaemia time during proctocolectomy in patients with UC. Resection specimens of 31 patients were available, five of which were from the colon and were therefore not considered. Non-inflamed $(n=21)$ and inflamed mucosa $(n=26)$ was dissected from freshly resected ileum $(n=26)$ of patients with $C D$, rinsed with iced saline, and snap frozen. Biopsy samples from the rectus abdominis muscle were taken at the beginning of laparotomy of patients with CD $(n=19)$ and snap frozen.

CONTROLS

Healthy age and sex matched subjects were used as controls for blood analysis $(n=65)$. Mucosa taken from small bowel of noncachectic patients undergoing right colectomy for local colonic tumours $(n=21)$ and muscle from patients undergoing laparotomy for various benign non-catabolic diseases $(n=18)$ (abdominal wall hernia $(n=3)$, aortic aneurysm $(n=6)$, hiatal hernia $(n=3)$, benign paraganglioma $(n=1)$, gastric ulcer $(n=1)$, mucocele of the appendix $(n=1)$, colon adenoma $(n=2)$, gallstones $(n=1)$ ) were used as controls.

BIOCHEMICAL ANALYSIS

Deproteinised plasma samples were subjected to amino acid analysis by HPLC (Biotronic LC 3000, Maintal, Germany). Repetitive calibration of amino acid analysis was performed every 7-10 samples within each series using a commercially available amino acid calibration standard (Type P-ANB, Eppendorf/Biotronic, Hamburg, Germany). Additionally, we used an aliquot of a frozen pooled plasma sample as a control sample. Plasma cysteine was determined spectrophotometrically (Ultrospec Plus, Pharmacia, Freiburg, Germany) at $412 \mathrm{~nm}$ as acid-soluble thiol using the reagent 5.5dithiobis-2-nitrobenzoate (DTNB) as described previously. ${ }^{33}$ Seven freshly prepared cysteine solutions with different concentrations were used as standards. Linearity of analysis 


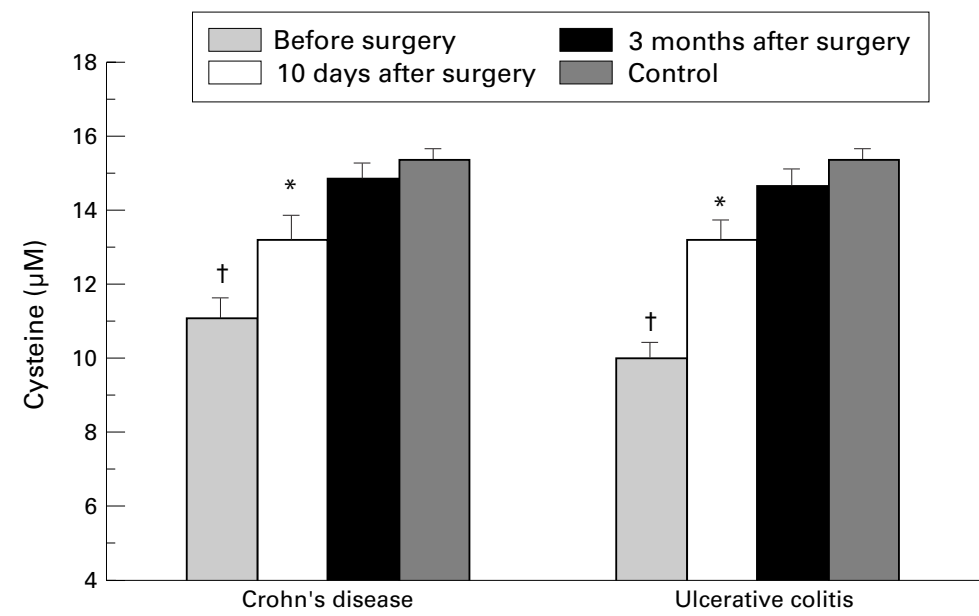

Figure 2 Fasting plasma cysteine levels in patients with Crohn's disease (n=33) and ulcerative colitis $(n=33)$ before surgery and 10 days and three months after complete resection of inflamed bowel. Bars represent mean (SE). Data were statistically analysed by the Student's $t$ test for unpaired samples including a Bonferroni's correction. ${ }^{\star} p<0.008$, $t p<10^{-4}$ versus controls $(n=65)$.

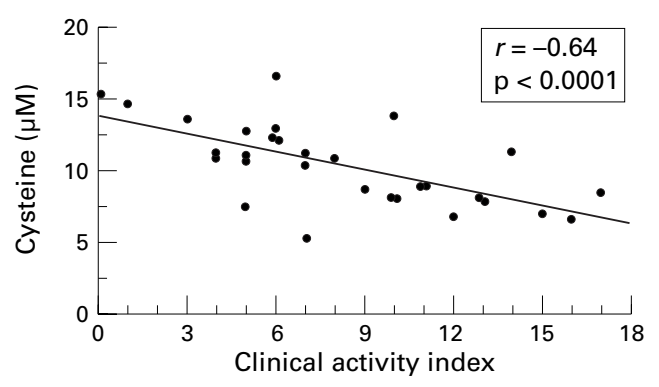

Figure 3 Linear regression analysis of preoperative plasma cysteine levels plotted against the individual clinical activity index according to Rachmilewitz in patients with ulcerative colitis.

was confirmed in each series. Pulverised tissue was homogenised in $2.5 \%$ SSA by sonification (Branson Sonifier 250, Branson Ultratronics, Danbury, USA) and centrifuged at $4^{\circ} \mathrm{C}$ (12000 $\mathrm{g}, 10$ minutes). For analysis of total GSH (tGSH) in mucosa, muscle, PBMC, and RBC the supernatant was assayed enzymatically as previously described with minor modifications. ${ }^{34}$ Briefly, $10 \mu \mathrm{l}$ supernatant of

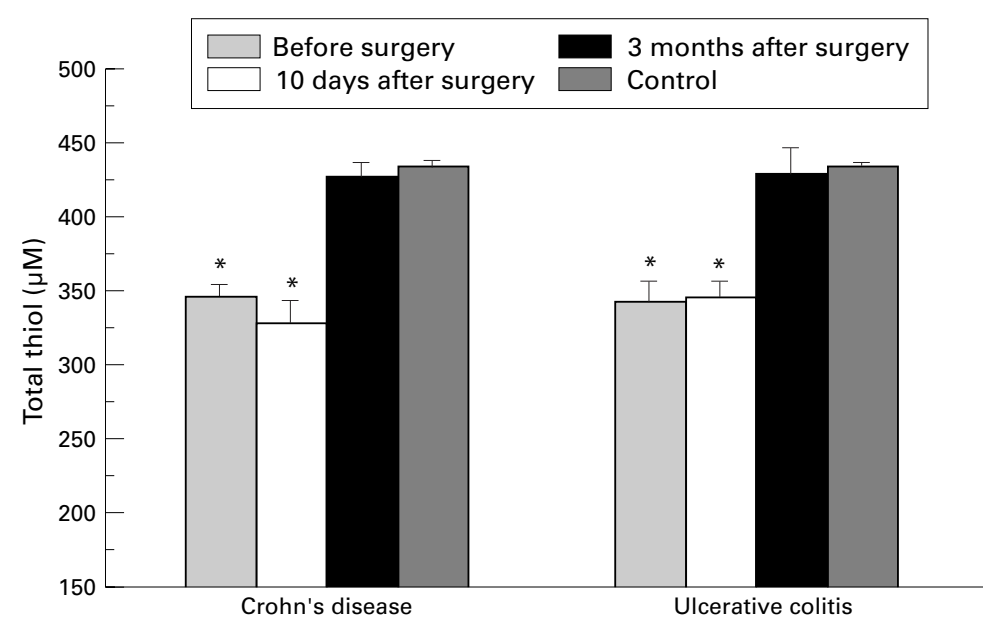

Figure 4 Total thiol levels in non-deproteinised plasma in patients with Crohn's disease $(n=33)$ and ulcerative colitis $(n=33)$ before surgery and 10 days and three months after complete resection of inflamed bowel. Values represent means (SE) and were statistically analysed by the Student's $t$ test for unpaired samples. According to Bonferroni's correction the level of significance was set at $p<0.008 .{ }^{\star} p<10^{-4}$ versus controls $(n=65)$. the relevant sample was mixed with $90 \mu \mathrm{SSA}$ $(2.5 \%), 6 \mu \mathrm{l}$ triethanolamine, $0.5 \mathrm{ml}$ buffer $\left(0.1 \mathrm{M} \mathrm{Na}_{2} \mathrm{HPO}_{4} ; 5 \mathrm{mM}\right.$ EDTA; $\left.\mathrm{pH} 7.5\right)$ and $0.2 \mathrm{ml} \mathrm{DTNB}(3 \mathrm{mM})$ and $1 \mathrm{U}$ yeast glutathione reductase (Sigma, Deisenhofen, Germany). Finally, $0.2 \mathrm{ml}$ NADPH $(1 \mathrm{mM})$ was added and the absorbance recorded at $41 \mathrm{~nm}$. The rate of reaction at $25^{\circ} \mathrm{C}$ was expressed as the change in absorbance per six minutes. GSSG was determined according to the method of Griffith ${ }^{35}$ after derivatisation of reduced GSH (rGSH) in $0.1 \mathrm{ml}$ supernatant with $2 \mu$ 2-vinylpyridine for 30 minutes at room temperature. Analysis then proceeded as described above. Nine freshly prepared solutions with different concentrations of GSH and GSSG, respectively, were used as standards to calculate the GSH/GSSG concentration in the tissue samples from a linear correlation between absorbance and concentration. The amount of $\mathrm{rGSH}$ was calculated as follows: $\mathrm{rGSH}=\mathrm{tGSH}-2 \times \mathrm{GSSG}$. Protein was determined by the Lowry method. ${ }^{36}$

For measurement of enzyme activity of $\gamma$ GCS mucosa samples were sonificated in $0.1 \mathrm{M}$ Tris- $\mathrm{HCl}$ buffer ( $\mathrm{pH} 8.0$ ) containing $0.15 \mathrm{M} \mathrm{KCl}, 20 \mathrm{mM} \mathrm{MgCl}_{2}$, and $2 \mathrm{mM}$ $\mathrm{Na}_{2}$ EDTA. Analysis was performed using a coupled enzyme procedure according to the method of Seelig. ${ }^{37}$ Enzyme activity in the reaction mixture was obtained from the decrease in absorbance of NADH at $340 \mathrm{~nm}$ $\left(37^{\circ} \mathrm{C}\right)$. For measurement of $\gamma$-glutamyl transferase $(\gamma \mathrm{GT})$ activity mucosa samples were sonificated in $0.4 \mathrm{ml}$ Tris- $\mathrm{HCl}$ buffer $(0.1 \mathrm{M}$, $\mathrm{pH}$ 8.25) and analysis was performed using a commercially available assay kit (Boehringer Mannheim, Mannheim, Germany). For both enzymes a linear increase in enzyme activity with increasing volumes of tissue homogenate and a linear correlation between absorbance and reaction time has been confirmed in a separate set of experiments. The protein content in the homogenate was determined according to Bradford's method. ${ }^{38}$

ANALYSIS OF DATA

Group comparison for statistical analysis was performed using the Student's $t$ test for unpaired samples (two tailed) after analysis of variance. The level of significance $(p<0.05)$ was corrected according to Bonferroni to allow for multiple comparisons. Results are presented as mean (SE).

\section{Results}

CONSTITUENT AMINO ACIDS OF GSH IN PLASMA AND INTESTINE

The constituent amino acids cysteine and its disulphide cystine, as well as glutamate and glycine were measured in plasma of patients with CD and UC preoperatively, and 10 days and three months postoperatively after resection of inflamed bowel. In both groups of IBD cysteine was found to be significantly decreased before the operation in the presence of intestinal inflammation compared with healthy control subjects (fig 2). Lowest cysteine levels were observed in patients with UC. Cysteine levels rapidly normalised early after removal of 
Table 1 Perioperative plasma concentrations $(\mu M)$ of GSH precursor amino acids in patients with IBD

\begin{tabular}{llll}
\hline & Cystine & Glutamate & Glycine \\
\hline Control (n=65) & $61.3(1.7)$ & $28.0(1.6)$ & $247(5)$ \\
Crohn's disease (n=33) & & & \\
$\quad$ Before surgery & $42.8(2.4)^{\star \star \star}$ & $32.3(3.3)$ & $254(14)$ \\
10 days after surgery & $56.0(3.0)$ & $36.2(2.8)^{\star \star}$ & $324(20)^{\star \star \star}$ \\
$\quad 3$ months after surgery & $52.7(2.8)^{\star \star}$ & $31.6(3.0)$ & $268(15)$ \\
Ulcerative colitis (n=33) & & & \\
$\quad$ Before surgery & $47.3(1.8)^{\star \star \star}$ & $36.7(3.2)^{\star \star}$ & $234(13)$ \\
10 days after surgery & $64.3(2.4)$ & $44.0(3.1)^{\star \star \star}$ & $327(19)^{\star \star \star}$ \\
3 months after surgery & $64.5(3.6)$ & $34.3(2.5)$ & $337(23)^{\star \star \star}$ \\
\hline
\end{tabular}

Values are expressed as mean (SE).

${ }^{\star \star} \mathrm{p}<0.01,{ }^{\star \star \star} \mathrm{p}<0.001 v$ healthy controls $(\mathrm{n}=65)$ (Student's $t$ test for unpaired samples, Bonferroni's correction).

Table 2 Concentration (nmol/mg protein) of GSH percursor amino acids in ileal mucosa from patients with Crohn's disease

\begin{tabular}{llll}
\hline & Cystine & Glutamate & Glycine \\
\hline Controls (n=21) & $0.92(0.13)$ & $39.7(3.4)$ & $33.0(2.9)$ \\
Crohn's disease & & & \\
$\quad$ Non-inflamed $(\mathrm{n}=21)$ & $0.80(0.14)$ & $37.1(2.6)$ & $30.2(2.0)$ \\
$\quad$ Inflamed $(\mathrm{n}=26)$ & $0.83(0.16)$ & $33.0(3.2)$ & $26.1(2.2)$ \\
\hline
\end{tabular}

Values are expressed as mean (SE). Data were analysed using the Student's $t$ test for unpaired samples. Significance was set at $\mathrm{p}<0.025$, according to Bonferroni's correction.

inflamed bowel and did not differ from controls three months after the operation in both IBD groups. Interestingly, a separate group of patients with CD without intestinal inflammation, but with manifestation of chronic perianal fistulas, had normal cysteine levels (13.9 (0.7) $\mu \mathrm{M}$ versus $15.3(0.4) \mu \mathrm{M}$ in controls; NS). In patients with UC low preoperative cysteine levels were closely correlated with a high clinical activity index (CAI) of Rachmilewitz $\left(r=0.64, \mathrm{p}<10^{-4}\right.$; fig 3$)$. Median cysteine levels in controls (CAI 0) and UC patients with low activity (CAI 1-6), moderate activity (CAI $7-12)$, and high activity (CAI $>12$ ) were 15.3 , $12.2,8.9$, and $7.9 \mu \mathrm{M}$, respectively. Such a correlation could not be established in CD patients using the CDAI index according to Best. Similarly, the total thiol content in deproteinised plasma in IBD, mainly representing protein sulfhydryls, was significantly reduced to the same extent in both CD and UC preoperatively (fig 4). Compared with cysteine, reduction of total thiol levels in plasma persisted early postoperatively but returned to normal values three months after removal of inflamed bowel.

Cystine levels were significantly decreased in both CD and UC patients in the presence of intestinal inflammation (table 1) but rapidly normalised early after the operation. While UC patients maintained high cystine levels three months after restorative proctocolectomy, plasma cystine concentrations dropped again below controls in patients with CD. Glycine levels did not differ from controls in both IBD groups before the operation. However, a long lasting increase was recorded in patients with UC after resection of inflamed bowel, whereas glycine levels initially increased early postoperatively in patients with CD and again reached preoperative values three months later. Glutamate, which is known to inhibit intracellular uptake of cystine competitively, ${ }^{25}$ was the only constituent amino acid found to be above control levels before the operation. We observed a $22 \%$ higher mean concentration of glutamate in patients with CD compared with controls; the $36 \%$ increase seen in patients with UC did not completely return to normal at the end of the observation period.

In contrast to the presented variations in plasma levels of precursor amino acids of GSH, mucosal concentrations of cystine, glutamate, and glycine consistently tended to be lower in the ileum of patients with CD by $10 \%, 17 \%$, and $21 \%$, respectively, compared with controls (table 2). Mucosal cystine concentrations did not parallel the pronounced decrease in plasma levels and mucosal uptake of glutamate was somewhat impaired despite high plasma levels in patients with $\mathrm{CD}$.

INTRACELLULAR GSH CONTENT IN PBMC, RBC, AND MUSCLE

Preoperative plasma analysis revealed a pattern of amino acids which suggests an impairment of GSH synthesis. However, analysing PBMC in the same CD patients we were able to demonstrate that intracellular rGSH and GSSG levels did not differ from controls irrespective of inflammation (table 3). Similar values, although slightly lower, were obtained in patients with UC. tGSH levels were even found to be increased in RBC in patients with CD compared with controls (13.8 (0.9) versus 9.6 $(0.5) \mathrm{nmol} / \mathrm{mg}$ protein; $\left.\mathrm{p}<10^{-4}\right)$. This finding was not associated with intestinal inflammation, as results were identical three months after removal of inflamed bowel (15.0 (1.0) $\mathrm{nmol} / \mathrm{mg}$ protein; $\mathrm{p}=10^{-6}$ versus controls). A normal redox status of glutathione in both PBMC and RBC, as defined by the ratio rGSH/GSSG, argues against considerable oxidative stress in the blood compartment in IBD. Similarly, tissue levels of rGSH in the muscle of patients with CD were not decreased despite plasma cyst(e)ine deficiency (11.6 (1.4) versus $13.3(1.6) \mathrm{nmol} / \mathrm{mg}$ protein in controls; NS). As GSSG in muscle was marginally above control values in patients with CD (0.91 (0.16)

Table 3 Glutathione status in peripheral blood mononuclear cells (PMBC) in IBD

\begin{tabular}{llll}
\hline & $r G S H$ (nmol/mg protein) & GSSG (nmol/mg protein) & rGSH/GSSG \\
\hline $\begin{array}{lll}\text { Control (n=65) (n) } \\
\text { Crohn's disease (n=3) }\end{array} \quad 22.8(1.2)$ & $2.05(0.11)$ & $12.2(0.8)$ \\
$\quad$ Before surgery & $23.0(2.0)$ & $2.06(0.17)$ & $14.7(2.0)$ \\
$\quad$ 10 days after surgery & $20.2(1.4)$ & $2.23(0.21)$ & $11.8(1.5)$ \\
$\quad$ months after surgery & $22.2(1.5)$ & $2.19(0.20)$ & $12.0(1.2)$ \\
Ulcerative colitis (n=33) & $19.5(1.3)$ & $2.25(0.24)$ & $11.5(1.3)$ \\
$\quad$ Before surgery & $17.9(1.3)$ & $2.43(0.25)$ & $10.0(1.2)$ \\
10 days after surgery & $20.7(1.1)$ & $2.30(0.20)$ & $11.0(1.1)$
\end{tabular}

Values are expressed as mean (SE). Data were analysed using the Student's $t$ test for unpaired samples. Significance was set at $\mathrm{p}<0.008$, according to Bonferroni's correction. 

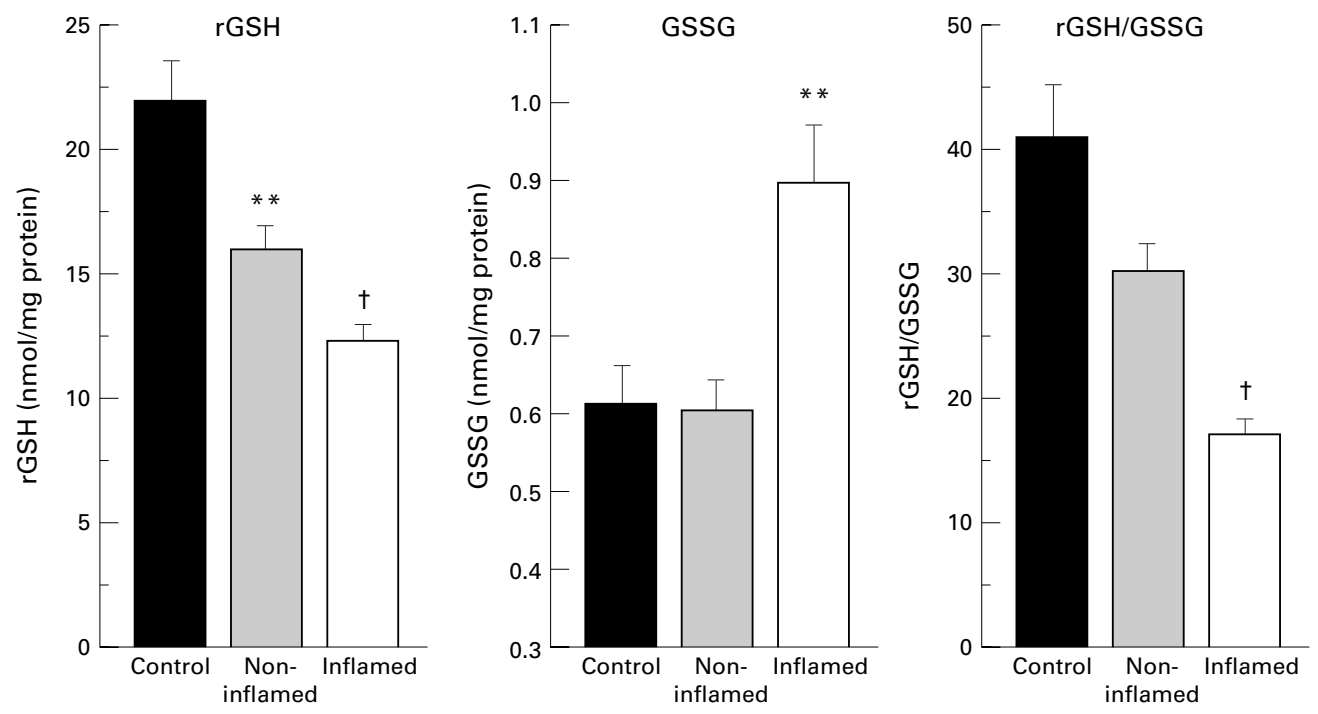

Figure 5 Pro-oxidant glutathione status in the mucosa of freshly resected ileum in patients with Crohn's disease. Reduced glutathione $(r G S H)$ is decreased in inflamed $(n=26)$ as well as non-inflamed $(n=21)$ mucosa in Crohn's disease. Oxidised glutathione (GSSG) is increased in areas of inflammation so that the redox status of glutathione, as defined by the ratio rGSH/GSSG, is heavily decreased in inflamed mucosa. Bars represent mean (SE). Statistical analysis was performed by the Student's $t$ test including a Bonferroni's correction. ${ }^{\star *} p<0.01,+p<10^{-4}$ versus controls $(n=21)$.

versus $0.74(0.16) \mathrm{nmol} / \mathrm{mg}$ protein; NS), the glutathione redox status tended to be decreased in this tissue but did not reach statistical significance (17.1 (2.3) versus 23.7 (2.9) in controls). In summary, deficiency of the sulphur-containing precursor amino acids of GSH in plasma does not result in an impairment of GSH synthesis in blood cells and muscle in IBD, irrespective of the presence or absence of intestinal inflammation.

GUT MUCOSAL GSH CONTENT

The finding of unimpaired GSH synthesis in the blood cells and muscle prompted us to investigate the GSH metabolism in inflamed and non-inflamed mucosa of the small bowel. Only patients with CD were considered for

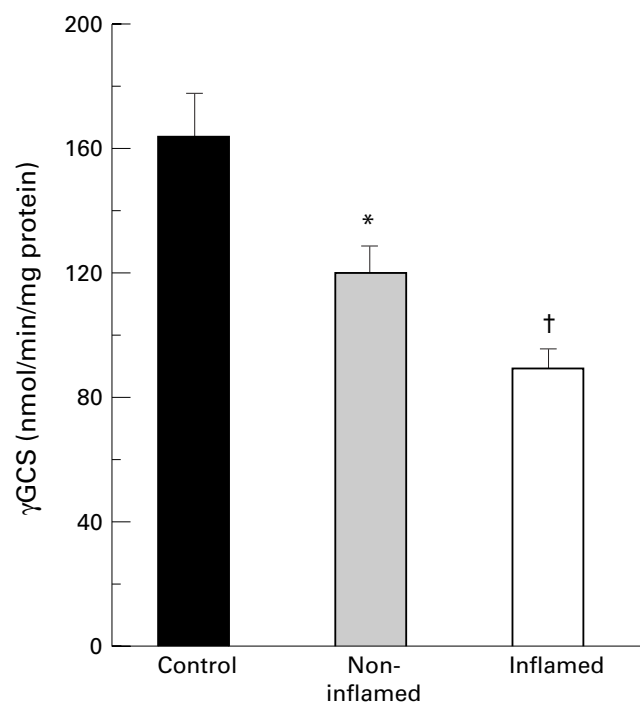

Figure 6 Decreased mucosal enzyme activity of $\gamma$-glutamylcysteine synthetase ( $\gamma$ GCS) in inflamed $(n=26)$ and non-inflamed $(n=21)$ ileum of patients with Crohn's disease. Bars represent mean (SE). Statistical analysis was performed by the Student's $t$ test for unpaired samples including a Bonferroni's correction. ${ }^{\star} p<0.025$, $t p<10^{-4}$ versus controls $(n=21)$. these investigations because the large bowel was subject to long ischaemia times during proctocolectomy in patients with UC. In contrast to results obtained in blood cells and muscle, mucosal rGSH was profoundly reduced by $24 \%$ and $38 \%$ in non-inflamed and inflamed mucosa, respectively (fig 5). Despite the shortage of rGSH, GSSG significantly increased with inflammation. Consequently, the GSH redox status (ratio rGSH/GSSG) dramatically decreased down to $42 \%$ in the inflamed ileum. This difference between patients with CD and controls is not simply the consequence of chronic steroid therapy as the GSH redox status in inflamed mucosa of patients receiving steroids $(n=13)$ was 18.1 (1.8) versus 16.0 (1.7) in patients without steroids $(n=13)(N S)$.

\section{GUT MUCOSAL $\gamma$ GCS ACTIVITY}

$\gamma$ GCS is the rate limiting enzyme in GSH synthesis and activity is negatively regulated via a feedback mechanism by the amount of GSH produced. ${ }^{24}$ In CD, however, we observed a profound decrease in $\gamma$ GCS enzyme activity despite GSH deficiency in inflamed and non-inflamed small bowel mucosa (fig 6). Chronic steroid therapy did not improve $\gamma$ GCS enzyme activity which was $95.2(12.1) \mathrm{nmol} /$ $\mathrm{min} / \mathrm{mg}$ protein in patients receiving steroids and $83.2(5.6) \mathrm{nmol} / \mathrm{min} / \mathrm{mg}$ protein in patients not receiving steroids (NS).

\section{GUT MUCOSAL $\gamma$ GT ACTIVITY}

$\gamma \mathrm{GT}$ is located at the luminal side of the brush border membrane of intestinal epithelial cells. It degrades luminal GSH and GSSG to $\gamma$-glutamyl amino acids that are transported to the cell where they are ultimately used for resynthesis of GSH (fig 1). ${ }^{28}$ Similarly to $\gamma$ GCS, $\gamma$ GT activity was considerably decreased in inflamed mucosa of the ileum compared with controls and was 17.7 (1.4) and $16.7(1.6) \mathrm{mU} / \mathrm{mg}$ protein in patients with and 


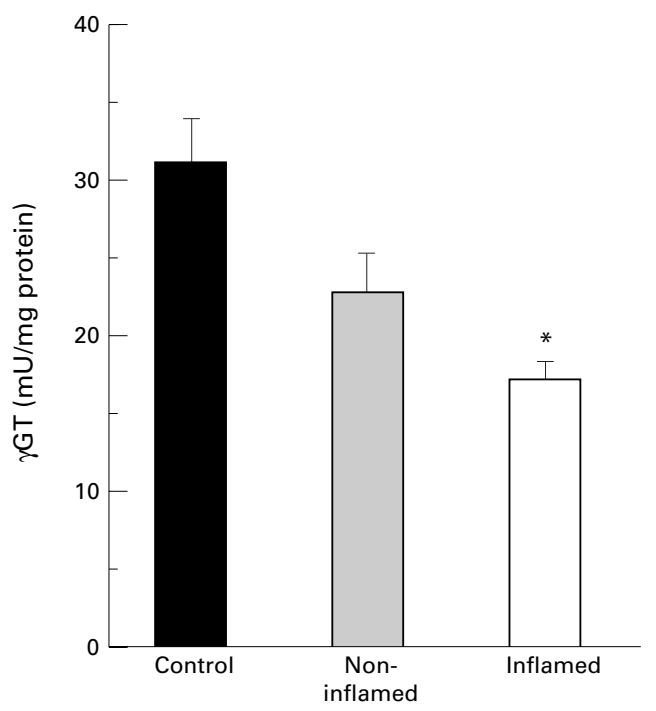

Figure 7 Decreased mucosal enzyme activity of $\gamma$-glutamyl transferase $(\gamma G T)$ in inflamed $(n=26)$ and non-inflamed $(n=21)$ ileum of patients with Crohn's disease. Bars represent mean (SE). Statistical analysis was performed by the Student's $t$ test for unpaired samples. According to Bonferroni's correction the level of significance was set at $p<0.025$. ${ }^{\star} p<10^{-4}$ versus controls $(n=21)$.

without steroids, respectively (NS). In noninflamed mucosa of patients with CD enzyme activity was intermediate between controls and inflamed tissue (fig 7).

\section{Discussion}

In this study we present a detailed analysis of gut mucosal GSH synthesis in patients with CD. Decreased activities of enzymes involved in GSH synthesis ( $\gamma \mathrm{GCS}, \gamma \mathrm{GT})$ predominantly contribute to intestinal GSH deficiency in both non-inflamed and inflamed ileum. Because the GSH level was shown to be normal or even increased in extraintestinal compartments, decreased availability of the sulphurcontaining constituent amino acids of GSH in plasma (but not in the mucosa) seems to be of minor importance. This impaired mucosal antioxidative capacity ultimately increases gut susceptibility to oxidative tissue injury. Despite intestinal rGSH deficiency, the GSSG level increased with inflammation resulting in a prooxidant shift of the mucosal redox balance of GSH (rGSH/GSSG) in CD. This could have major implications on intestinal immune functions in IBD as pro-oxidant mechanisms are known to be involved in the activation of the transcription factor $\mathrm{NF \kappa B}$, thereby mediating the transcription of several immunologically relevant cytokines and cell adhesion molecules. ${ }^{39}$

Cysteine and its disulphide cystine are known to be limiting substrates in GSH biosynthesis ${ }^{22}{ }^{27}$ and therefore generate a strong influence on intracellular GSH stores. ${ }^{33} 340$ Reduced plasma cysteine and cystine levels are not unique to patients with inflammatory bowel disease and could also be demonstrated in other catabolic diseases such as HIV infection $^{33}$ and advanced malignant tumour growth $^{41}{ }^{42}$ in conjunction with a reduction in intracellular GSH levels in PBMC. In patients with IBD cysteine deficiency seems to be a consequence and not a primary mechanism of relapsing inflammation because (1) cysteine and cystine levels rapidly normalise after removal of inflamed bowel in both $\mathrm{CD}$ and UC, (2) patients with CD without intestinal inflammation but with manifestations of chronic perianal fistulas have normal cysteine values, (3) UC, which usually involves larger bowel segments, is accompanied by lower cysteine levels than $\mathrm{CD}$, and (4) preoperative cysteine levels significantly correlate with the clinical disease activity in patients with UC. Plasma cysteine levels could be decreased due to ongoing inflammation for several reasons. The sulfhydryl amino acid cysteine is a potent scavenger of hydroxyl radicals and could be readily consumed due to oxidative stress in the inflamed gut. In support of this explanation is our finding that the decrease in total thiol content in plasma, mainly representing protein sulfhydryls, is also associated with inflammation in both CD and UC. Alternatively, hypochlorite that is produced from hydrogen peroxide and chloride via the myeloperoxidase reaction in neutrophils was shown to be present in the mucosa of rectal biopsy samples in patients with IBD. ${ }^{43}$ The reaction of hypochlorite with ammonia yields monochloramine. Both hypochlorite and monochloramine avidly degrade cysteine ${ }^{44}$ and could account for cysteine deficiency. Inversely, the therapeutically effective drug 5 -aminosalicylic acid is a potent antioxidant, ${ }^{14}$ inhibits neutrophil production of hypochlorite and monochloramine, ${ }^{44-46}$ and protects cysteine from degradation by these two toxic compounds. ${ }^{44}$ Supplementation of cysteine or $\mathrm{N}$-acetyl-L-cysteine might have beneficial effects on disease activity due to its radical scavenging activity and as it helps to replenish intracellular GSH stores. Oral application of cysteine has recently been shown to ameliorate tissue injury in experimental colitis. ${ }^{47}$

Although the pattern of precursor amino acid, as described in the present study, suggests impairment of GSH synthesis in IBD, our results do not argue for overall inhibition of GSH synthesis. Among the compartments analysed we could show a decrease of GSH in the intestine, but not in blood cells and muscle. The reason for the increased GSH content in $\mathrm{RBC}$ is presently not known but has also been observed in tumour bearing mice in the presence of decreased plasma cyst(e)ine levels. ${ }^{48}$ It is unlikely that plasma deficiency of cyst(e)ine is due to avid amino acid uptake into RBC for subsequent GSH synthesis as plasma cyst(e)ine levels normalise postoperatively, although increased GSH levels persist in RBC. In cultured cells cystine is taken up by the membrane amino acid transport system $x_{c}$ that imports cystine in exchange for glutamate. ${ }^{49}$ In vitro this transport system can be induced by glutathione deficiency or oxidative stress. ${ }^{50}$ Provided that such a mechanism is active in vivo, induction of the transport system could explain why mucosal levels of cystine were not found to be decreased to the same extent below control levels as were plasma levels and, 
inversely, why glutamate levels were decreased in the ileum. In the face of a normal or even increased GSH concentration in extraintestinal compartments, decreased mucosal glutathione levels are not solely due to insufficient uptake of precursor amino acids from plasma. Rather, it has to be assumed that intestinal GSH synthesis is impaired. The gut is highly dependent on GSH synthesis as has been shown in mice. Treatment with L-buthionineSR-sulphoximine, a specific inhibitor of $\gamma$ GCS, rapidly decreased mucosal content of GSH down to $60 \%$ of control values within 30 minutes. ${ }^{23}$ In the present study we could demonstrate a $45 \%$ downregulation of mucosal $\gamma$ GCS activity in patients with CD that could easily account for decreased GSH levels in the gut mucosa. $\gamma \mathrm{GCS}$, the rate limiting enzyme in GSH synthesis, is negatively regulated via a feedback mechanism by which high concentrations of the end product GSH inhibit the enzyme. $^{24}$ Obviously, the physiological feedback regulation of $\gamma$ GCS by GSH is not operative in patients with CD despite mucosal GSH deficiency. Feedback inhibition of $\gamma \mathrm{GCS}$ by GSH can be blocked by a high concentration of intracellular glutamate that competes with $\mathrm{GSH}$ at the regulatory site of the enzyme. ${ }^{24}$ However, such a mechanism can also be excluded as mucosal glutamate levels were shown to be below controls in CD.

Loss of intracellular GSH could also cause mucosal GSH deficiency. GSSG formed by oxidative stress is either reduced to GSH via the glutathione reductase-catalysed reaction or, when produced in excess amounts, is transported from the cells to the blood in order to maintain equilibrium levels of GSH and GSSG. $^{50}$ Alternatively, GSSG reacts with protein sulfhydryls via a mixed disulphide reaction. ${ }^{51}$ The net result of both mechanisms is a loss of intracellular free GSH. Another drain on the intracellular pool of GSH is the glutathione S-transferase reaction which catalyses the conjugation of glutathione with a large number of electrophilic compounds. Although glutathione S-transferase was reported to be present in the intestine, ${ }^{52}$ our laboratory could provide evidence for a decrease in glutathione S-transferase activity in the gut mucosa in CD (unpublished observation).

Intestinal epithelial cells were shown to contain high levels of $\gamma \mathrm{GT}$ activity predominantly at the villous tips. ${ }^{53}$ We could demonstrate a significant decrease in $\gamma \mathrm{GT}$ activity in the inflamed ileum of patients with CD. Consequently, degradation products of luminal GSH cannot be made available to the intestine for resynthesis of GSH to maintain physiological $\mathrm{GSH}$ levels. However, previous studies in vascularly perfused rat small intestine and in vivo studies in rats with specific inhibition of $\gamma$ GT by acivicin showed that the salvage pathway via $\gamma \mathrm{GT}$ is of minor importance. ${ }^{12}{ }^{54}$ This finding is supported by studies from our laboratory which have shown that the decrease in colonic GSH levels in CD is identical to the ileum, although $\gamma \mathrm{GT}$ activity in the colon was found to be only $31 \%$ of the activity in the ileum and remains unchanged in CD (unpub- lished data). Thus the present study provides evidence that several factors relevant to GSH synthesis in gut mucosa are impaired; the reduced blood supply of the precursor amino acids cysteine and cystine in conjunction with downregulation of mucosal $\gamma \mathrm{GT}$ activity and, most important, profound reduction in $\gamma$ GCS enzyme activity argue for insufficient GSH synthesis as a primary mechanism for mucosal GSH deficiency.

GSH is essential for the function and structural integrity of the gut; GSH-deficient mice show severe degeneration of the jejunum and colonic mucosa and were found to have body weight loss and diarrhoea. ${ }^{23}$ Oral administration of a mixture of constituent amino acids (L-glutamate, L-cysteine, L-glycine) was shown not to be effective in increasing gut mucosal GSH levels. ${ }^{12}{ }^{23}$ In contrast, high amounts of GSH result in supply of GSH to the lower gastrointestinal tract and, probably due to uptake of intact GSH from the lumen across the brush border membrane, thus provide a direct means of raising GSH levels in the mucosa of the jejunum, ileum and colon. ${ }^{12}{ }^{23}{ }^{30}$ Based on the profound impairment of GSH synthesis as demonstrated in the present study, GSH supplementation might be of therapeutic value in IBD.

The study was financially supported by the DKFZ project 072006

1 Mahida YR, Wu KC, Jewell DP. Respiratory burst activity of intestinal macrophages in normal and inflammatory bowel disease. Gut 1989;30:1362-70.

2 Williams JG. Phagocytes, toxic oxygen metabolites and inflammatory bowel disease: implications for treatment. Ann R Coll Surg Engl 1990;72:253-62.

3 Grisham MB, Granger DN. Neutrophil-mediated mucosal injury. Role of reactive oxygen metabolites. Dig Dis Sci 1988;33:6S-15S.

4 Weiss SJ. Tissue destruction by neutrophils. $N$ Engl f Med 1989;320:365-76.

5 Williams JG, Hughes LE, Hallert MB. Toxic oxygen metabolite production by circulating phagocytic cells in metabolite production by circulating phagocytic

6 Grisham MB, Volkmer C, Tso P, et al. Metabolism of trinitrobenzene sulfonic acid by rat colon produces reactive oxygen species. Gastroenterology 1991;101:540-7.

7 Millar AD, Rampton DS, Chander CL, et al. Evaluating the antioxidant potential of new treatments for inflammatory bowel disease using a rat model of colitis. Gut 1996;39: $407-15$

8 Keshavarazian A, Sedghi S, Kanovsky J, et al. Excessive production of reactive oxygen metabolites by inflamed colon: analysis by chemiluminescence probe. Gastroenterology 1992;103:177-85.

9 Grisham MB, MacDermott RP, Deitch EA. Oxidant defense mechanisms in the human colon. Inflammation 1990;6:669-80.

10 Babbs C. Oxygen radicals in ulcerative colitis. Free Radic Biol Med 1992;13:169-81.

11 van der Vliet A, Bast A. Role of reactive oxygen species in intestinal diseases. Free Radic Biol Med 1992;12:499-513.

12 Iantomasi T, Marraccini P, Favilli F, et al. Glutathione metabolism in Crohn's disease. Biochem Med Metabol Biol metabolism in Cro 1 .

13 Lih-Brody L, Powell SR, Collier KP, et al. Increased oxidative stress and decreased antioxidant defenses in mucosa of inflammatory bowel disease. Dig Dis Sci 1996;41:2078-86.

14 Ahnfelt-Ronne I, Nielssen OH. The anti-inflammatory moiety of sulfasalzine, 5-aminosalicylic acid, is a radical scavanger. Agents Actions 1987;21:191-4.

15 Ahnfelt-Ronne I, Nielsen OH, Christensen A, et al. Clinical evidence supporting the radical scavenger mechanism of 5-aminosalicylic acid. Gastroenterology 1990;98:1162-9.

16 Emerit J, Pelletier S, Likforman J, et al. Phase II trial of superoxide dismutase (CuZn SOD) in the treatment of Crohn's disease. Free Radic Res Commun 1991;12-13(Pt 2):563-9.

17 Niwa Y, Somiay K, Michelson AM, et al. Effect of liposomal-encapsulated superoxide dismutase on active oxygen-related disorders. Free Radic Res Commun 1985;1: oxygen-relat.

18 Simmonds NJ, Blake DR, Rampton DS. The effects of aminosalicylates and potential new drugs on mucosal reactive oxygen metabolite production in ulcerative colitis. Gut 1992;33(suppl):S7. 
19 Tamai H, Levin S, Gaginella TS. Induction of colitis in rats by 2-2'-azobis(2-amidinopropane) dihydrochloride. Inflammation 1992;16:69-81.

20 Grisham MB. Oxidants and free radicals in inflammatory bowel disease. Lancet 1994;344:859-61.

21 Gross V, Arndt H, Andus T, et al. Free radicals in inflammatory bowel diseases. Pathophysiology and therapeutic implications. Hepatogastroenterol 1994;41:320-7.

22 Meister A, Anderson ME. Glutathione. Ann Rev Biochem 1983;52:711-60.

23 Martensson J, Jain A, Meister A. Glutathione is required for intestinal function. Proc Natl Acad Sci USA 1990;87:17159.

24 Richman P, Meister A. Regulation of gamma-glutamylcysteine synthetase by nonallosteric feedback inhibition by glutathione. Biol Chem 1975;250:1422-6.

25 Eck H-P, Dröge W. Influence of the extracellular glutamate concentration on the intracellular cyst(e)ine concentration in macrophages and on the capacity to release cysteine. Biol Chem Hoppe-Seyler 1989;370:109-13.

26 Bannai S, Kitamura E. Transport interaction of L-cystine and L-glutamate in human diploid fibroblasts in culture. $f$

27 Meister A. Selective modification of glutathione metabolism. Science 1983;220:472-7.

28 Vincenzini MT, Favilli F, Iantomasi T. Intestinal uptake and transmembrane transport systems of intact GSH: characteristics and possible biological role. Biochim Biophys Acto 1992;1113:13-23.

29 Hagen TM, Wierzbicka GT, Sillau AH, et al. Bioavailability of dietary glutathione: effect on plasma concentrations. $A m$ f Physiol 1990;259:G524-9.

30 Hagen TM, Wierzbicka GT, Bowman BB, et al. Fate of dietary glutathione: disposition in the gastrointestinal tract. Am $\mathcal{F}$ Physiol 1990;259:G530-5.

31 Best WR, Becktel JM, Singleton JW, et al. Development of a Crohn's disease activity index. National Cooperative Crohn's Disease Study. Gastroenterology 1976;70:439-44.

32 Rachmilewitz D. Coated mesalazine (5-aminosalicylic acid) versus sulphasalzine in the treatment of active ulcerative versus sulphasalzine in the treatment of active
colitis: a randomised trial. $B M \mathcal{F} 1989 ; 298: 82-6$.

33 Eck H-P, Gmünder H, Hartmann M, et al. Low concentrations of acid-soluble thiol (cysteine) in the blood plasma of HIV-1-infected patients. Biol Chem Hoppe-Seyler 1989;370 $101-8$

34 Gmünder H, Eck HP, Benninghoff $\mathrm{B}$, et al. Macrophages regulate intracellular glutathione levels of lymphocytes. Evidence for an immunoregulatory role of cysteine. Cell Immunol 1990;129:32-46.

35 Griffith OW. Determination of glutathione and glutathione disulfide using glutathione reductase and 2-vinyl-pyridine. Anal Biochem 1980;106:207-12.

36 Lowry OH, Rosenbrough WJ, Farr AL, et al. Protein measurement with the folin phenol reagent. F Biol Chem 1951;193:265-75

37 Seelig GF, Meister A. Glutathione biosynthesis; $\gamma$-glutamylcysteine synthetase from rat kidney. Methods $\gamma$-glutamylcysteine synth
Enzymol 1985;13:379-90.
38 Bradford MM. A rapid and sensitive method for the quantification of microgram quantities of protein utilizing the principle of protein-dye binding. Anal Biochem 1976;72: $248-54$

39 Siebenlist U, Franzoso G, Brown K. Structure, regulation and function of NF-кB. Annu Rev Cell Biol 1994;10:405-55.

40 Ishii T, Sugita Y, Bannai S. Regulation of glutathione in mouse spleen lymphocytes by transport of cysteine. $f \mathrm{Cell}$ Physiol 1987;133:330-6.

41 Eck H-P, Drings P, Dröge W. Plasma glutamate levels, lymphocyte reactivity and death rate in patients with bronchial carcinoma. F Cancer Res Clin Oncol 1989;115:571-4.

42 Hack V, Schmid D, Breitkreutz R, et al. Cystine levels, cystine infection and senescence. FASEB F 1997;11:84-92.

43 Simmonds NJ, Allen RE, Stevens TR, et al. Chemiluminescence assay of mucosal reactive oxygen metabolites in inflammatory bowel disease. Gastroenterology 1992;103. 186-96.

44 Tamai H, Kachur JF, Grisham MB, et al. Scavenging effect of 5 -aminosalicylic acid on neutrophil-derived oxidants. Possible contribution to the mechanism of action in inflammatory bowel disease. Biochem Pharmacol 1991;41:1001-6.

45 Williams JG, Hallett MB. The reaction of 5-amino-salicylic acid with hypochlorite. Implications for the mode of action in inflammatory bowel disease. Biochem Pharmacol 1989; 38: $149-54$.

46 Dallegri F, Ottonello L, Ballestrero A, et al. Cytoprotection against neutrophil derived hypochlorous acid: a potential mechanism for the therapeutic action of 5-aminosalicylic acid in ulcerative colitis. Gut 1990;31:184-6.

47 Karmeli F, Rachmilewitz D. Cysteine and methionine: a novel modality to ameliorate tissue injury in experimental colitis [abstract]. Gastroenterology 1997;112:A1009.

48 Hack V, Gross A, Kinscherf R, et al. Abnormal glutathione and sulfate levels after interleukin 6 treatment and in tumor-induced cachexia. FASEB f 1996;10:1219-26.

49 Bannai S. Exchange of cystine and glutamate across plasm membrane of human fibroblasts. F Biol Chem 1986;261 2256-62.

50 Deneke SM, Fanburg BL. Regulation of cellular glutathione. Am f Physiol 1989;257:L163-73.

51 DeLucia AJ, Mustafa MG, Hussain MZ, et al. Ozone interaction with rodent lung. III. Oxidation of reduced glutathione and formation of mixed disulfides between protein and nonprotein sulfhydryls. F Clin Invest 1975;55:794-802.

52 Peters WHM, Roelofs MMJ, Nagengast FM, et al. Human intestinal glutathione S-transferases. Biochem f 1989;257: 471-6.

53 Cornell JS, Meister A. Glutathione and $\gamma$-glutamyl cycle enzymes in crypt and villus tip cells of

54 Hagen TM, Jones DP. Transepithelial transport of glutathione in vascularly perfused small intestine of rat. $A m \mathcal{F}$ Physiol 1987;252:G607-13. 